\title{
GA GSA-based Energy Efficient Pegasis Protocol for WSN
}

\author{
Suraj Srivastava* and Dinesh Grover \\ Department of Research, Innovation and Consultancy \\ IKG Punjab Technical University, Jalandhar, Punjab, India \\ *surajsriengg@gmail.com,dineshgrover@yahoo.com
}

\begin{abstract}
WSN is termed as wireless sensor network that consists of very large number of nodes that are connected with each other. These nodes are used to sense large amount of distinct data, interact with each other, and are computationally capable. The sensor nodes deployed in wireless network are light in weight and have constrained battery power. Hence, energy is a main problem of wireless sensor networks. This paper proposes a method for energy minimization with efficient clustering using GSA algorithm in Wireless Sensor Network. To compute cluster head election probability this work uses Genetic Algorithm method. Various parameters are used to evaluate the performance of the proposed technique. Experimental results demonstrate that the proposed technique outperforms existing techniques by various parameters.
\end{abstract}

Keywords: wireless sensor network, pegasis, genetic algorithm, gravitational search algorithm, energy efficiency, cluster head

\section{Introduction}

WSN is a collection of wireless nodes with limited power that can be mobile or stationary and are positioned randomly in a dynamically changing environment. The routing techniques choice is a crucial decision for the efficient transport of the packets to their destination. Moreover, in such networks, the applied routing method should make sure the minimum of the electricity intake and as a result maximization of the lifetime of the network [1].

Recently, advances in wireless communication and virtual electronics have led to the development of Wireless Sensor Network (WSN). WSN has been implemented in many fields which include military, clinical treatment and enterprise management. Therefore, research of techniques to prolong the network lifetime is an important and challenging problem, which is also the focus of designing the WSN routing protocol [2].

Wireless Sensor Networks (WSN) typically includes low energy nodes having sensors, computation unit, a battery module and transmission module. The only source of energy is the battery, that's not going to be replaced. Hence, power conservation has become one of the most crucial challenges in WSNs [3].

The microcontroller is designed to consume extremely-low energy since the attached battery is typically non-replaceable once the sensor nodes are deployed. In addition, the dimensions of the battery are also restrained on account that sensor nodes are required to be small. It also has limited storage capacity [4].

Energy consumption of a sensor node has a tremendous impact at the lifetime of WSNs. The electricity of a WSN can be stored by means of making use of exclusive techniques. An efficient routing protocol can help stabilize the energy consumption

Received (May 15, 2018), Review Result (July 10, 2018), Accepted (July 15, 2018)

* Corresponding Author 
among WSN nodes. Given the equal hardware conditions, it is able to help extend the lifetime of WSN [5].

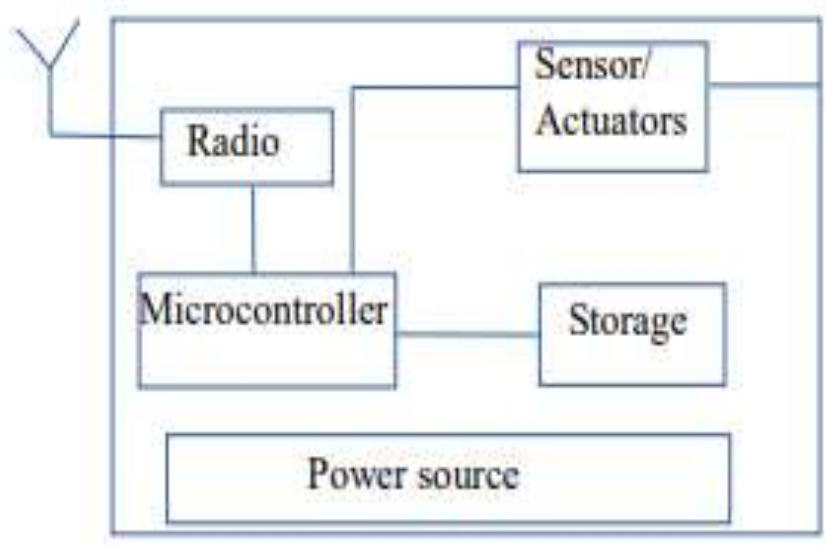

Figure 1. Typical Sensor Node [4]

\section{Prior Work}

Sharma et al., mentioned and evaluated pegasis with simulator NS2 for performance difficulty. Routing algorithm decides which route will be accompanied through packets for conversation between sender and receiver. Wireless Sensor Network includes nodes with confined energy source and low bandwidth. Optimal route for verbal exchange saves extra quantity of power of the network. PEGASIS protocol is based totally on hierarchical network structure proposed for Wireless Sensor Network [6].

Guo et al., proposed a routing protocol based totally on the PEGASIS protocol however the use of an advanced ant colony set of rules as opposed to the grasping algorithm to assemble the chain. Compared with the unique PEGASIS, PEGant, could gain a global optimization. It made a series that made the direction more even-disbursed and the full square of transmission distance a good deal much less. Moreover, inside the design procedure, the strength element has been taken into account, which brings approximately a stability of power consumption between nodes. In every round of transmission, according to the modern power of every node, a frontrunner is chosen to without delay to the BS. Simulation results have shown that the proposed protocol appreciably prolongs the network lifetime [7].

Linping et al., proposed a new set of rules-PDCH, at the bases of PEGASIS to make each nodes load balanced and increase the network lifetime. Protocol PEGASIS is based totally on the chain structure, each chain have most effective one cluster head, it's far in rate with every node's receiving and sending messages that belong to this chain, the cluster head consumes massive power and the instances of each spherical growing [8].

Chauhan et al., proposed a contrast of LEACH and PEGASIS protocol that is meant to stabilize the power intake of the complete network and increase the life of the network. Clustering is used for the network lifetime and it's far very important technique in Mobile AD Hoc Networks. The scheme is used by the cluster head node which performs a totally crucial role inside the transmitting packet procedure from one cluster to the opposite or nearest node. The energy resource of each sensor node is restrained within the cluster. Minimizing electricity dissipation and maximizing network lifetime is a difficult task within the design of routing protocols for sensor networks [9].

Watkar et al., proposed one-of-a-kind energy stable routing protocols for WSNs in comparison to specific protocols of WSN, that maximized network lifetime with the aid of balancing the weight as far as possible. Wireless sensor network (WSN) is a system 
composed of a large variety of low-fenergy micro-sensors. This community is used to collect and send numerous forms of messages to a base station (BS). WSN includes lowprice nodes with restricted battery energy, and the battery replacement isn't clean for WSN with heaps of physically embedded nodes, which means power efficient routing protocol ought to be employed to offer a protracted network lifestyles time. The life of Wireless Sensor Networks (WSN) is crucial. To accomplish the said purpose, not only overall electricity consumption needs to be decreased, however, additionally there is a requirement to balance the WSN load [10].

Darji et al., suggested another routing protocol that is having energy harvested from surroundings in preference to batteries. Traditional routing protocols which include LEACH, PEGASIS, TEEN and many others are not suitable for the Energy HarvestingWireless Sensing element Networks (EH-WSN). Requirement is that WSN should have low power consumption. Machine Learning algorithms can be used for minimizing power consumption. [11].

Garg et al., explored the competing problems of power consumption efficiency in wi-fi sensor networks. To acquire this, they have taken into consideration Gravitational Search algorithm (GSA) inside the selection of Cluster Head (CH) in any such manner in order that its power is used uniformly with behind schedule disintegration of community. For this reason, the LEACH algorithm random clustering technique has been changed by GSA clustering. The GSA based LEACH protocol has been as compared with random LEACH, Max Energy LEACH and k-manner set of rules. Total network machine lifetimes are determined the use of an expansion of small probabilities of the available device nodes. The performance of GSA cluster primarily based routing protocol suggests a few differences by varying lifestyles sample amongst nodes and quantity of useless nodes. Max Energy LEACH carry out better in community disintegration criterion however it provides much less network lifetime compared to GSA based totally LEACH [12].

Parvin et al., proposed novel technique of facts aggregation mechanism using cellular sink nodes in Wireless sensor community to enhance the lifetime of the sensor nodes. The sink nodes are used in WSNs to acquire the sensed data from the Cluster Heads; those are located a long way from the Base Station. Network lifetime is increased with the assistance of sink nodes by removing the overload of Cluster Heads. In the proposed work, nodes are allowed to shape as clusters and no Cluster Head election is being executed. Whereas, the operation of Cluster Heads is being taken care by way of the newly brought cell sink nodes to perform accumulating the sensed statistics, aggregating it and verbal exchange operations. Thus the lifetime of the sensor nodes has increased significantly [13].

\section{The Proposed Work}

This section discusses about methodology of the proposed technique. This research work proposes a method for energy minimization with efficient clustering using GSA algorithm in Wireless Sensor Network. PEGASIS is chain based method for hierarchical network architecture primarily based on greedy algorithm. Nodes interact with most effective near neighbor nodes in PEGASIS. The center concept of this proposed technique is to use topology control to exploit spatial node redundancy in formation of chain. Minimum value of GA and GSA is computed to check if there is any node that lies within range of closest distance or not. The steps to implement the proposed technique are:

1. Start residual node reduction, total energy consumption, packet delivery ratio, network lifetime for the network.

2. A hello message is transmitted in whole network to achieve network information like identity of nodes alive, distance of a node from base station, \& distance between nodes.

3. Search farthest node from base station \& set it as end node.

4. Calculate $S_{\mathrm{NR}}$ 
- Sort distance among node in sensor field.

- Choose one percent of minimum distances.

- Calculate average (AVG).

- $\mathrm{S}_{\mathrm{NR}}=\operatorname{Min}(\mathrm{GA}, \mathrm{GSA}) * \mathrm{AVG}$

5. Search node with distance less than $\mathrm{S}_{\mathrm{NR}}$ from end node.

6. Turn off chosen nodes.

7. Search for active node having minimum distance from end node.

8. Join it with chain and set it as next end node.

9. Continue these steps until all nodes are traversed.

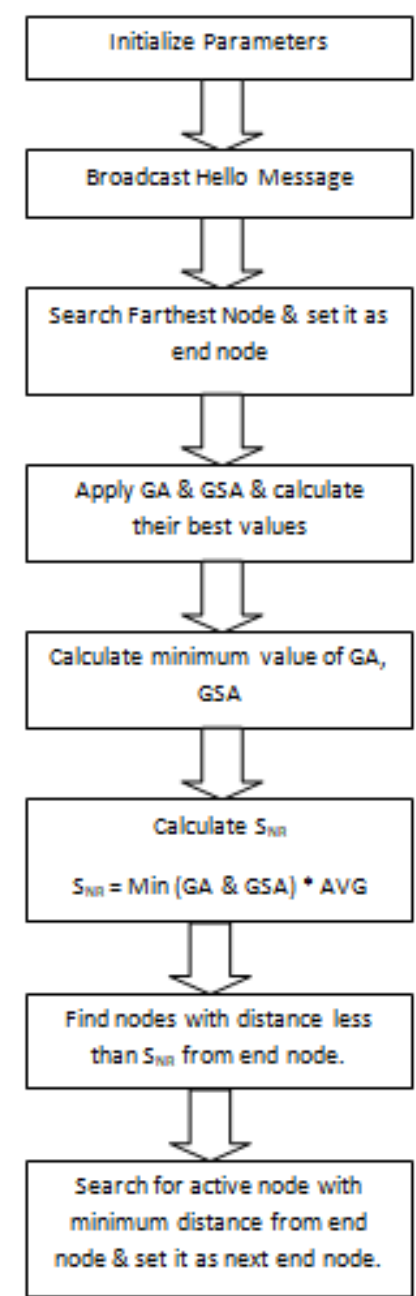

Figure 2. Flowchart of Proposed Technique

\subsection{Genetic Algorithm}

In GA nodes we initialize as population by opting genetic behavior, best fitness function result as best population, to calculate fitness value. Three steps (crossover, mutation, selection) are repeated for specific number of generations to find local solution. It is best in case when we have two options for a solution.

- Chromosome: It is a set of genes that consists the solution in the form of genes. For e.g., 98150 is a chromosome value then 9,8,1,5 and 0 are its genes.

- Population: The total number of individuals or chromosome with same number of genes. 
- Fitness: It is a value that assigned to an individual based on readiness of an individual to provide solution. Greater the fitness value, more appropriate solution will be acquired.

- Fitness function: It is an application oriented objective function which assigns fitness value to the individual.

- Crossover: Taking two fit individuals and then performs intermingling process to generate new two individuals.

- Mutation: It is a method of changing random genes in an individual value.

- Selection: The method of choosing individual to generate the new generation.

\subsection{Gravitational Search Algorithm}

GSA is a physical phenomenon based upon Law of Gravity and Law of Motion to locate global optimized solution. Working of ACO and GSA is quite comparable but computation features are definitely different. ACO selects one quality solution among two different entities while GSA provides all over one great solution among population. The Gravitational Search Algorithm considered as an isolated system for small artificial world of masses which obeying the Newton Law of gravitation and motion.

\section{Experiments and Results}

\subsection{Comparison based on Alive Nodes}

This section presents the comparison of the proposed modified Pegasis GA GSA with Pegasis and pegasis with fuzzy on the basis of alive nodes.

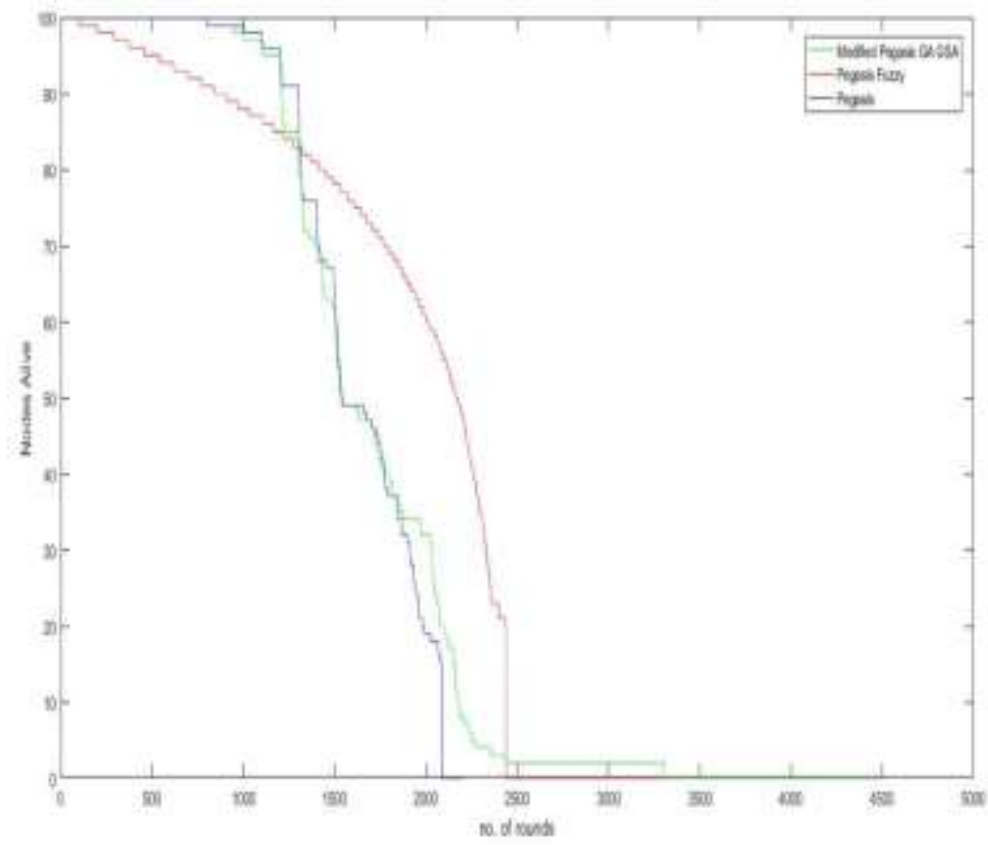

Figure 3. Comparison on basis of Alive Nodes of Proposed Technique with Existing Techniques

Above figure shows the comparison of the proposed technique modified pegasis with GA GSA with the existing technique pegasis and pegasis with fuzzy based on alive nodes. It is clear from the above figure that the proposed technique has more number of alive nodes as compared to the existing techniques. 


\subsection{Comparison based on Dead Nodes}

This section presents the comparison of the proposed modified Pegasis with GA GSA and Pegasis with fuzzy and Pegasis on the basis of dead nodes.

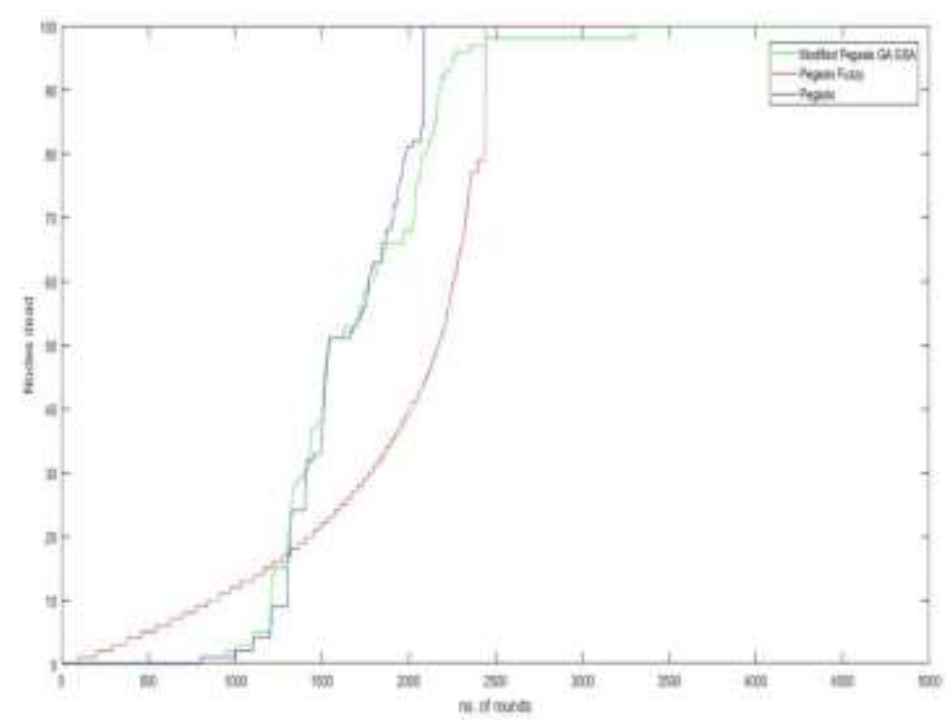

Figure 4. Comparison on basis of Dead Nodes of Proposed Technique with Existing Techniques

Above figure shows the comparison of the proposed technique modified pegasis with GA GSA with the existing technique pegasis and pegasis with fuzzy based on dead nodes. It is clear from the above figure that the proposed technique has less dead nodes as compared to the existing techniques.

\subsection{Comparison based on Packets Sent to Base Station}

This section presents the comparison of the proposed modified Pegasis with GA GSA with Pegasis \& pegasis with fuzzy on the basis of dead nodes.

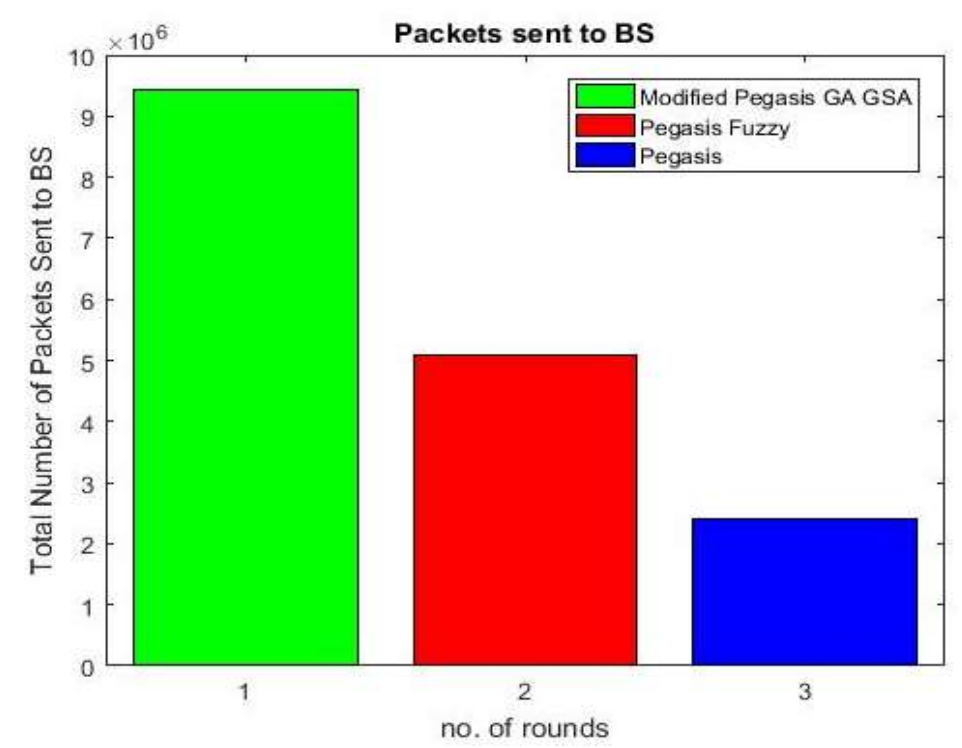

Figure 5. Comparison on basis of Packets Sent to base Station of Proposed Technique with Existing Techniques 
Above figure shows the comparison of the proposed technique modified pegasis with GA GSA with the existing technique pegasis and pegasis with fuzzy based on packets sent to base station. It is clear from the above figure that the proposed technique sends more packets to base station as compared to the existing techniques.

\section{Conclusion}

This paper proposes a method for energy minimization with efficient clustering using GSA algorithm in Wireless Sensor Network. To compute cluster head election probability this work use Genetic Algorithm method. Results are evaluated on the basis of number of alive nodes, number of dead nodes, and number of packets sent to base station. Proposed technique modifies pegasis with GA GSA as compared with existing techniques pegasis and pegasis with fuzzy on basis of the above mentioned parameters. Results show that proposed technique gives more number of alive nodes, and packet sent to base station whereas less number of dead nodes as compared to existing techniques.

\section{References}

[1] N. A. Pantazis, S. A. Nikolidakis and D. D. Vergados, "Energy-Efficient Routing Protocols in Wireless Sensor Networks: A Survey", IEEE, Communications Surveys \& Tutorials, vol. 15, no. 2, (2013), pp. 551-590.

[2] W. Guo, W. Zhang and G. Lu, "PEGASIS Protocol in Wireless Sensor Network Based on an Improved Ant Colony Algorithm", IEEE, International Workshop on Education Technology and Computer Science, (2010), pp. 64-67.

[3] M. Pramanick, P. Basak, C. Chowdhury and S. Neogy, "Analysis of Energy Efficient Wireless Sensor Networks Routing Schemes", IEEE, International Conference of Emerging Applications of Information Technology, (2014), pp. 379-384.

[4] M. Loganathan, T. Sabapathy, N. Hafizah Ghazali, R. Badlishah Ahmad and M. Nasrun Osman, "Energy efficient routing protocols for wireless sensor networks: comparison and future directions", (2017), pp. 1-7.

[5] J. Yan, M. Zhou and Z. Ding, "Recent Advances in Energy-Efficient Routing Protocols for Wireless Sensor Networks: A Review”, IEEE, (2016), pp. 5673-5686.

[6] I. Sharma, R. Singh and M. Khurana, "Performance Evaluation of PEGASIS Protocol for WSN using NS2", IEEE, International Conference on Advances in Computer Engineering and Applications, (2015), pp. 926-929.

[7] W. Guo, W. Zhang and G. Lu, "PEGASIS Protocol in Wireless Sensor Network Based on an Improved Ant Colony Algorithm", IEEE, International Workshop on Education Technology and Computer Science, (2010), pp. 64-67.

[8] W. Linping, C. Zhen, B. Wu and W. ZuJeng, "Improved algorithm of PEGASIS protocol introducing double cluster heads in wireless sensor network", IEEE, International Conference on Computer, Mechatronics, Control and Electronic Engineering, (2010), pp. 148-151.

[9] T. Chauhan and M. Nayyer, "A Technical Review on Energy Efficient Protocol based on PEGASIS and LEACH", International Journal on Recent and Innovation Trends in Computing and Communication, vol. 7, no. 7, (2016), pp. 303-307.

[10] S. Watkar, B. G. Hogade and H. Satam, "Review Paper on Energy Efficient Protocol in Wireless Sensor Network", Int. Journal of Engineering Research and Applications, vol. 5, no. 3, (2015), pp. 107-110.

[11] H. Darji and H. B. Shah, "Genetic Algorithm for Energy Harvesting-Wireless Sensor Networks", IEEE, International Conference On Recent Trends in Electronics Information Communication Technology, (2016), pp. 1398-1402.

[12] P. Garg, R. Rani and G. Singh, "Achieving Energy Efficiency in WSN using GSA", International Journal of Advanced Research in Computer Science and Software Engineering, vol. 4, no. 4, (2014), pp. $168-172$.

[13] J. Rejina Parvin and C. Vasanthanayaki, "Mobile Sink Nodes for Energy Efficient Wireless Sensor Networks using Gravitational Search Algorithm", Australian Journal of Basic and Applied Sciences, (2015), pp. 171-181. 


\section{Authors}

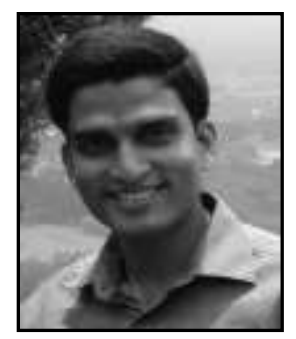

Suraj Srivastava received his BTECH in Computer Science and Engineering from Prasad Institute of Technology Jaunpur, Uttar Pradesh, India in 2007 and MTECH in Computer Science and Engineering from Dr B R Ambedkar National Institute of Technology, Jalandhar, Punjab, India in the year 2009. He is currently pursuing $\mathrm{PhD}$ in the Department of Research, Innovation and Consultancy, IKG Punjab Technical University, Punjab, India. His professional research activity lies in the field of Wireless Sensor Networks and Data mining.

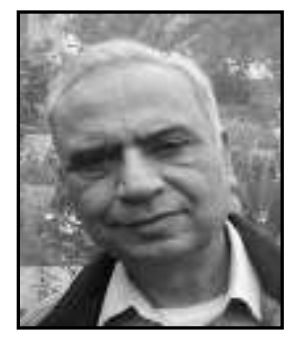

Dinesh Grover is alumni of the PEC University, Chandigarh and the University of Roorkee (Now IIT Roorkee), Roorkee, India. He has worked in various academic and administrative positions at PAU Ludhiana, LLRIET, Moga and affiliated colleges of IKG PTU, Jalandhar. Presently he is working as Adjunct Professor in the department of Computer Science and Engineering, GNDEC, Ludhiana. His major areas of interest are broadband optical wireless communication systems, networks, data mining, wireless sensor networks and computer communication. He has published many research papers in the International/National Journals/Conferences. 